\title{
8/10 HLA Match
}

National Cancer Institute

\section{Source}

National Cancer Institute. 8/10 HLA Match. NCI Thesaurus. Code C158474.

Degree of HLA match is 8/10 for HLA-A, -B, -C, -DRB1, and -DQB1 loci. 